\title{
Genotype frequencies of the $A B C C 11$ gene in 2000-3000-year-old human bones from the Epi-Jomon and Jomon sites in Hokkaido, Japan
}

\author{
Hisako KAZUTA ${ }^{1}$, Takehiro SATO ${ }^{1}$, Yukio DODO ${ }^{2}$, Hirofumi MATSUMURA ${ }^{3}$, Tetsuya AMANO ${ }^{4}$, \\ Hajime ISHIDA ${ }^{5}$, Ryuichi MASUDA ${ }^{1 *}$ \\ ${ }^{1}$ Graduate School of Science, Hokkaido University, Sapporo 060-0810, Japan \\ ${ }^{2}$ Tohoku University School of Medicine, Sendai 980-8575, Japan \\ ${ }^{3}$ Sapporo Medical University, Sapporo 060-8556, Japan \\ ${ }^{4}$ Hokkaido University Museum, Sapporo 060-0810, Japan \\ ${ }^{5}$ Faculty of Medicine, University of the Ryukyus, Nishihara 903-0215, Japan
}

Received 16 April 2010; accepted 2 August 2010

\begin{abstract}
In order to investigate further the allele frequencies of the adenosine triphosphate-binding cassette sub-family $\mathrm{C} 11$ ( $A B C C 11)$ gene, which determines earwax phenotypes, among ancient populations in Hokkaido, Japan, the single-nucleotide polymorphism and the $27 \mathrm{bp}$ deletion in the $A B C C 11$ gene were analyzed in ancient DNA extracted from 38 Epi-Jomon, 24 Jomon, and one Satsumon specimens excavated from various archaeological sites in Hokkaido. Of the specimens analyzed, 10 EpiJomon and three Jomon specimens were successfully genotyped. One homozygote for the $27 \mathrm{bp}$ deletion was found first in one Epi-Jomon specimen. Including previously reported data on the Okhotsk people and modern Ainu, exact tests of population differentiation showed that allele frequencies of the $A B C C 11$ gene between the Jomon people and Ainu were statistically significantly different, whereas those between the Jomon and Epi-Jomon people and those between the Epi-Jomon people and Ainu were not significantly different. This result indicates that the Ainu, direct descendants of the Jomon people, were genetically affected by populations who possessed high frequencies of allele A (recessive dry allele) of the $A B C C 11$ locus after the Jomon period.
\end{abstract}

Key words: $A B C C 11$, Ancient DNA, Jomon people, Epi-Jomon people, Okhotsk people

\section{Introduction}

Human earwax phenotypes consist of the dominant wet type and the recessive dry type, and are controlled by a single autosomal locus (Matsunaga, 1962). Recently, Yoshiura et al. (2006) reported that a single nucleotide polymorphism (SNP), $538 \mathrm{G} \rightarrow \mathrm{A}$ ( $\mathrm{rs} 17833931)$, in the adenosine triphoshate-binding cassette sub-family C11 (ABCC11) gene exon 4 , determines earwax phenotypes, where allele $\mathrm{G}$ is dominant and allele $\mathrm{A}$ is recessive. In addition, the $27 \mathrm{bp}$ deletion located in the $A B C C 11$ gene exon 29 leads allele $\mathrm{G}$ to lose its function as the dominant allele. Because the frequencies of allele A show a north-south and east-west downward gradient with a peak in northeastern Asia during Homo sapiens migration, it is considered that the mutation $(\mathrm{G} \rightarrow \mathrm{A})$ originated in northeastern Asia. Kitano et al. (2008) reported that the SNP in the $A B C C 11$ gene is a useful marker providing ancestry information on human populations, because the allele frequencies of the $A B C C 11$ gene are markedly different among various ethnic populations.

* Correspondence to: Ryuichi Masuda, Department of Natural History Sciences, Faculty of Science, Hokkaido University, Sapporo 060-0810, Japan.

E-mail: masudary@ees.hokudai.ac.jp

Published online 1 October 2010

in J-STAGE (www.jstage.jst.go.jp) DOI: 10.1537/ase.100416
On Hokkaido Island, northern Japan (Figure 1), cultures different from Honshu Island (largest main island in the Japanese archipelago) have developed. After the Jomon period (about 12000-2400 BP), the Yayoi culture with rice agriculture (3rd century BC-3rd century $\mathrm{AD}$ ) developed in Honshu, whereas the Epi-Jomon (3rd century BC-7th century AD), Satsumon (8th-14th centuries: Amano, 2003a) and Ainu cultures developed as direct descendants of the Jomon people in Hokkaido, instead of the Yayoi culture in Honshu (Dodo and Kawakubo, 2002). Moreover, the Okhotsk culture developed almost allopatrically around the coastal regions of the Okhotsk Sea, including northern and eastern Hokkaido, during the 5th-13th centuries (Amano, 2003b). Morphological (Yamaguchi, 1974, 1981; Ishida, 1988; Kozintsev, 1990, 1992; Komesu et al., 2008) and mitochondrial DNA (mtDNA) studies (Sato et al., 2007, 2009a) reported that the characteristics of the Okhotsk people were similar to those of the people currently living around the lower regions of the Amur River, such as the Ulchi, Nivkhi, and Negidal. At present, it is reasonable to consider that the Okhotsk people could have originated from common ancestors to the modern local people living around the lower regions of the Amur River.

Based on archaeological studies, the custom that the skulls of brown bears were frequently enshrined in pit houses of the Okhotsk culture is considered to be the origin of the bearsending ceremony of the Ainu culture (Utagawa, 2002). 

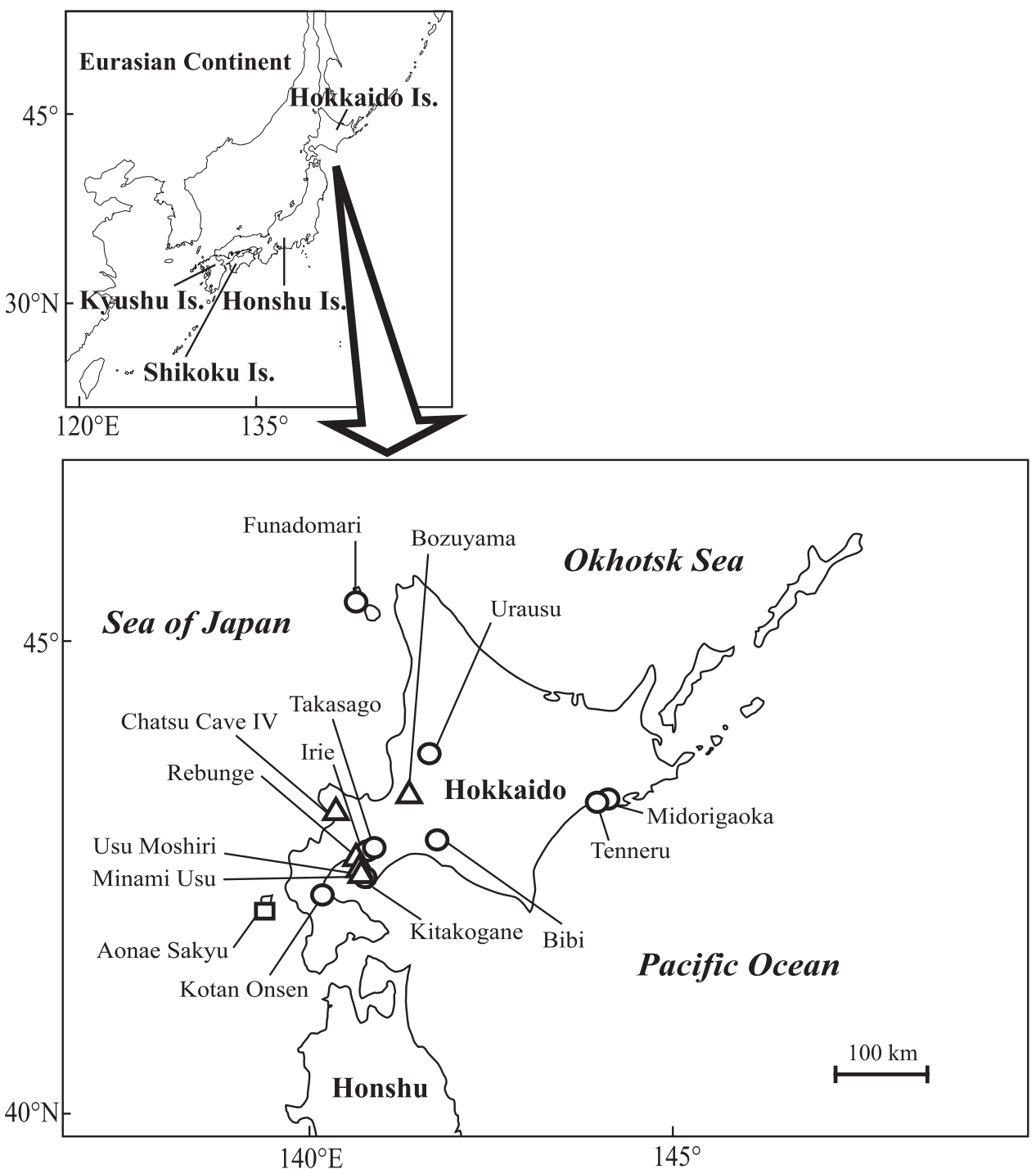

Figure 1. Geographical locations of archaeological sites where bone specimens analyzed in the present study were excavated. Circles, triangles and squares indicate archaeological sites of the Jomon, Epi-Jomon and Satsumon cultures, respectively.

Amano (2003b) reported that earthenware and pit houses at the end of the Okhotsk period seemed to have been strongly influenced by the Satsumon culture. In addition, ancient mtDNA analyses of the Okhotsk people (Sato et al., 2007, 2009a) revealed that haplogroup $Y$, which has not been identified from Jomon and Epi-Jomon specimens, was shared by the Okhotsk people at high frequency and by the Ainu at lower frequency. These findings suggest that the Okhotsk people merged with direct descendants of the Jomon people, resulting in the establishment of the Ainu.

The genetic characteristics of mtDNA of ancient populations in Hokkaido have been investigated (Adachi et al., 2006, 2009; Sato et al., 2007, 2009a). By contrast, it is extremely difficult to analyze ancient nuclear DNA, because it rarely survives in ancient human bone remains due to the smaller number of copies than exist for mtDNA molecules. Although mtDNA analysis provides particular information on genetic characteristics due to its high polymorphism, its sequences show only information on maternal lineage; therefore, information on biparentally inherited autosomal genes is necessary for further understanding of the genetic structures of ancient populations.

For autosomal DNA of the Jomon/Epi-Jomon and Okhotsk people, Sato et al. (2009b) analyzed allele frequencies of the $A B C C 11$ gene, and reported that the frequencies of recessive allele A increased in the order Jomon, Ainu and Okhotsk. In addition, they suggested that gene flow occurred from the Okhotsk people to the Jomon lineage; however, Sato et al. (2009b) succeeded in analyzing only one EpiJomon specimen, and information on the $A B C C 11$ gene alleles of the Epi-Jomon people is still lacking. The $A B C C 11$ gene determining earwax phenotypes is a particular gene providing information on the phenotypes of ancient people. The frequency of wet earwax in the Ainu is higher than among other northeastern Asian populations (Yoshiura et al., 2006). This high frequency of wet earwax in the Ainu is 
considered to have resulted from allele frequencies of the Jomon people in Hokkaido. Therefore, further analysis of the $A B C C 11$ gene could provide new information for verification of the hypothesis on the migration and history of ancient people.

In the present study, the SNP and the $27 \mathrm{bp}$ deletion in the $A B C C 11$ gene of additional Epi-Jomon and Jomon specimens from additional archaeological sites of Hokkaido were analyzed. Combining the data obtained in the present study and the previously reported data (Sato et al., 2009b), the allele frequencies were compared with those of modern Asian people (Yoshiura et al., 2006) to discuss gene flow among the Jomon, Epi-Jomon, Okhotsk people and Ainu, as previously shown by mtDNA data (Horai et al., 1996; Adachi et al., 2006, 2009; Sato et al., 2007, 2009a).

\section{Materials and Methods}

\section{Sample collection}

To determine the genotypes of the $A B C C 11$ gene of the ancient peoples of Hokkaido, 24 skeletal remains of the Jomon people, 38 of the Epi-Jomon people, and one of the Satsumon people, excavated from 15 archaeological sites (Figure 1), were analyzed. The skeletal remains were preserved at Tohoku University School of Medicine and Sapporo Medical University. To avoid duplicate analyses of remains from identical individuals, parts in the same positions of bones or bones from different graves within one archaeological site were used.

\section{DNA extraction}

Total DNA was extracted from skulls, mandibles, ribs, humeri, metacarpal bones, phalanges, coxal bones, femurs, tibiae, fibulae, tali, tarsal bones, and metatarsal bones of ancient skeletal remains excavated from archaeological sites. To avoid contamination of surface external DNA, each bone piece was soaked in sodium hypochlorite solution $(8.5-13.5 \% \mathrm{Cl}$, Nacalai) for $5 \mathrm{~min}$, rinsed with DNase-/ RNase-free distilled water, and air-dried. The bones were powdered using a dental drill. The DNA extraction methods for bone powder were carried out according to the methods of Masuda et al. (2001) and Sato et al. (2007, 2009a, b, 2010).

Approximately $0.2-0.5 \mathrm{~g}$ powder per specimen was decalcified overnight with $30 \mathrm{ml}$ of $0.5 \mathrm{M}$ ethylenediamine tetraacetic acid (EDTA; Nippongene) in a $50 \mathrm{ml}$ plastic tube with rotation at room temperature. The decalcified samples were suspended overnight in $5 \mathrm{ml}$ of $0.5 \mathrm{M}$ EDTA containing $100 \mu \mathrm{l}$ of $10 \mathrm{mg} / \mathrm{ml}$ proteinase $\mathrm{K}$ at $37^{\circ} \mathrm{C}$ with rotation. The solution was extracted using the phenol-chloroform extraction method (phenol/chloroform/isoamylalcohol 25:24:1: Sambrook et al., 1989). The DNA extracts were concentrated into approximately $200 \mu \mathrm{T}$ TE buffer with VivaSpin 6 Concentrators (Sartorius) and used for the subsequent polymerase chain reaction (PCR).

\section{Multiplex APLP analysis}

Using multiplex APLP analysis (Kitano et al., 2008), the SNP (rs178822931) and 27 bp deletion of the $A B C C 11$ gene were analyzed. An aliquot $(1 \mu \mathrm{l})$ of the DNA extract was used as a PCR template. PCR amplifications were carried out in reaction mixtures of $20 \mu \mathrm{l}$ containing reagents of the Multiplex PCR Kit (Qiagen), each primer of optimum concentration (Kitano et al., 2008), $0.4 \mu \mathrm{g} / \mu \mathrm{l}$ bovine serum albumin (BSA, Boehringer), and distilled water. The PCR conditions were $95^{\circ} \mathrm{C}$ for $15 \mathrm{~min}$, followed by 40 cycles of $95^{\circ} \mathrm{C}$ for $30 \mathrm{~s}, 60^{\circ} \mathrm{C}$ for $3 \mathrm{~min}, 72^{\circ} \mathrm{C}$ for $90 \mathrm{~s}$; and $72^{\circ} \mathrm{C}$ for $10 \mathrm{~min}$. An aliquot $(10 \mu \mathrm{l})$ of the PCR product was electrophoresed in polyacrylamide gel $(10 \% \mathrm{~T}, 5 \% \mathrm{C})$ containing $375 \mathrm{mM}$ Tris- $\mathrm{NaOH}$ buffer ( $\mathrm{pH} 8.9$ ) with running buffer (12.5 mM Tris, $96 \mathrm{mM}$ glycine, $\mathrm{pH}$ 8.3). The DNA bands were detected with an ultraviolet illuminator after staining with ethidium bromide. Because ancient DNA may have suffered post-mortem damage, three PCR amplifications from each sample were made to assess reproducibility of the results. Samples that did not show reproducibility were excluded from subsequent analysis.

\section{Contamination precautions}

To avoid contamination, the following standard contamination precautions were taken. Experiments from DNA extraction to pre-PCR were conducted on a clean bench. Gloves, face masks, laboratory coats, disposable filter-plugged pipette tips, and disposable tubes were used. The clean bench and instruments were treated with DNA-AWAY (Molecular BioProducts) before and after they were used. When DNA extraction and PCR were conducted, negative extraction controls and negative PCR controls were used.

\section{Data analysis}

To check for contamination and allelic dropout, HardyWeinberg equilibrium was tested for each population analyzed in the present study with GENEPOP 4.0 (Raymond and Rousset, 1995). Allele frequencies of the Jomon and Epi-Jomon people were compared with the previously reported data from the Okhotsk people (Sato et al., 2009b), Ainu, Nagasaki, Okinawa, Yonaguni, Han, and Korean (Yoshiura et al., 2006). Exact tests of population differentiation among the Jomon, Epi-Jomon, Okhotsk, and Ainu were carried out with ARLEQUIN 3.11 (Excoffier et al., 2005).

\section{Results and Discussion}

In the present study, DNA fragments, including the SNP (rs17822931) and the 27 bp deletion region, were successfully PCR-amplified and genotyped from three of 24 Jomon specimens and from ten of 38 Epi-Jomon specimens (Table 1). In particular, one homozygote of the 27 bp deletion was identified for the first time from an ancient bone specimen (EPJ-5). Among the ancient people of Hokkaido examined in the previous study (Sato et al., 2009b), this deletion was not found. Yoshiura et al. (2006) reported that the $27 \mathrm{bp}$ deletion was detected in only one of 334 modern Japanese, indicating that it is very rare in Japan. The finding of such a rare deletion in this Epi-Jomon specimen shows that the allele including the deletion already existed in Hokkaido during the Epi-Jomon period. Because the allele G and A frequencies of 18 Jomon, 1 Epi-Jomon and 31 Okhotsk specimens as ancient DNA data were reported by Sato et al. (2009b), we combined them and the allele frequency data obtained in the present study for statistical analysis (Table 2). 
Table 1. The ABCC11genotypes of the Jomon and Epi-Jomon people

\begin{tabular}{lllll}
\hline \multirow{2}{*}{ Specimen no. $\begin{array}{c}\text { Archaeological } \\
\text { site }\end{array}$} & \multicolumn{2}{c}{ Genotypes } & \\
\cline { 3 - 3 } & & rs17822931 & Deletion $^{\text {a }}$ & \\
\hline Jomon & & & & \\
JM-1 & Takasago & G/A & W/W & wet \\
JM-2 & Midorigaoka & G/G & W/W & wet \\
JM-3 & Bibi & G/G & W/W & wet \\
Epi-Jomon & & & & \\
EPJ-1 & Usumoshiri & A/A & W/W & dry \\
EPJ-2 & Usumoshiri & G/G & W/W & wet \\
EPJ-3 & Usumoshiri & A/A & W/W & dry \\
EPJ-4 & Usumoshiri & G/A & W/W & wet \\
EPJ-5 & Usumoshiri & G/G & $\Delta 27 / \Delta 27$ & dry \\
EPJ-6 & Usumoshiri & G/G & W/W & wet \\
EPJ-7 & Usumoshiri & A/A & W/W & dry \\
EPJ-8 & Chatu Cave IV & G/A & W/W & wet \\
EPJ-9 & Minami Usu & A/A & W/W & dry \\
EPJ-10 & Chatu Cave IV & A/A & W/W & dry \\
\hline
\end{tabular}

${ }^{\mathrm{a}} \mathrm{W}$ indicates the wild type, and $\Delta 27$ indicates the $27 \mathrm{bp}$ deletion.

The exact test showed that no deviation from HardyWeinberg equilibrium was observed in the Jomon people $(P>0.05)$. Concerning the Epi-Jomon people, the deviation from Hardy-Weinberg equilibrium was not observed for the SNP $(P>0.05)$, but was observed for the $27 \mathrm{bp}$ deletion. The latter deviation could have been caused by the existence of one homozygote of the deletion in the Epi-Jomon specimens.

Allele frequencies of the ancient people of Hokkaido were compared with the previously reported data from modern East Asian populations (Table 2, Figure 2): 58 Ainu, 126 Nagasaki, 58 Okinawa, 30 Yonaguni, 52 Han Chinese, and 99 Korean (Yoshiura et al., 2006). The frequencies of recessive allele A in the Jomon and the Epi-Jomon people were 0.476 and 0.591 , respectively. The frequencies of allele $\mathrm{A}$ increased in the order Jomon, Epi-Jomon, Ainu (0.759), and
Table 2. Frequencies of allele A among East Asian population

\begin{tabular}{lccl}
\hline Population & $\begin{array}{c}\text { No. of } \\
\text { samples }\end{array}$ & $\begin{array}{c}\text { Frequency of } \\
\text { allele A }\end{array}$ & \multicolumn{1}{c}{ Citation } \\
\hline Jomon & 21 & 0.476 & $\begin{array}{l}\text { Present study } \\
\text { Sato et al. (2009b) }\end{array}$ \\
Epi-Jomon & 11 & 0.591 & $\begin{array}{l}\text { Present study } \\
\text { Sato et al. (2009b) } \\
\text { Ainu }\end{array}$ \\
Okhotsk & 58 & 0.759 & Yoshiura et al. (2006) \\
Nagasaki & 126 & 0.839 & Sato et al. (2009b) \\
Okinawa & 58 & 0.829 & Yoshiura et al. (2006) \\
Yonaguni & 30 & 0.733 & Yoshiura et al. (2006) \\
Han Chinese & 52 & 0.683 & Yoshiura et al. (2006) \\
Korean & 99 & 1.000 & Yoshiura et al. (2006) \\
\hline
\end{tabular}

Okhotsk people (0.839). Exact tests of population differentiation showed that allele frequencies of the $A B C C 11$ gene between the Jomon and Okhotsk people, between the Jomon people and Ainu, and between the Epi-Jomon and Okhotsk people were significantly different $(P<0.05)$ (Table 3$)$. On the other hand, allele frequencies between the Jomon and Epi-Jomon people, between the Epi-Jomon people and Ainu, and between the Okhotsk people and Ainu were not significantly different $(P>0.05)$ (Table 3$)$.

Evidence of the bear-sending ceremony, which has been maintained in the Ainu culture, was found at archaeological sites of the Okhotsk culture (Utagawa, 2002). The earthenware and pit houses at the end of the Okhotsk period seem to be strongly influenced by the Satsumon culture (Amano, 2003b). In addition, mtDNA analyses indicated that haplogroup Y, which has not been found in the Jomon and EpiJomon people in Hokkaido, was identified in the Okhotsk people at higher frequencies (Sato et al., 2007, 2009a). Haplogroup Y was also found from the Ainu at a relatively lower frequency (Horai et al., 1996). These three findings suggest that gene flow occurred from the Okhotsk people to the

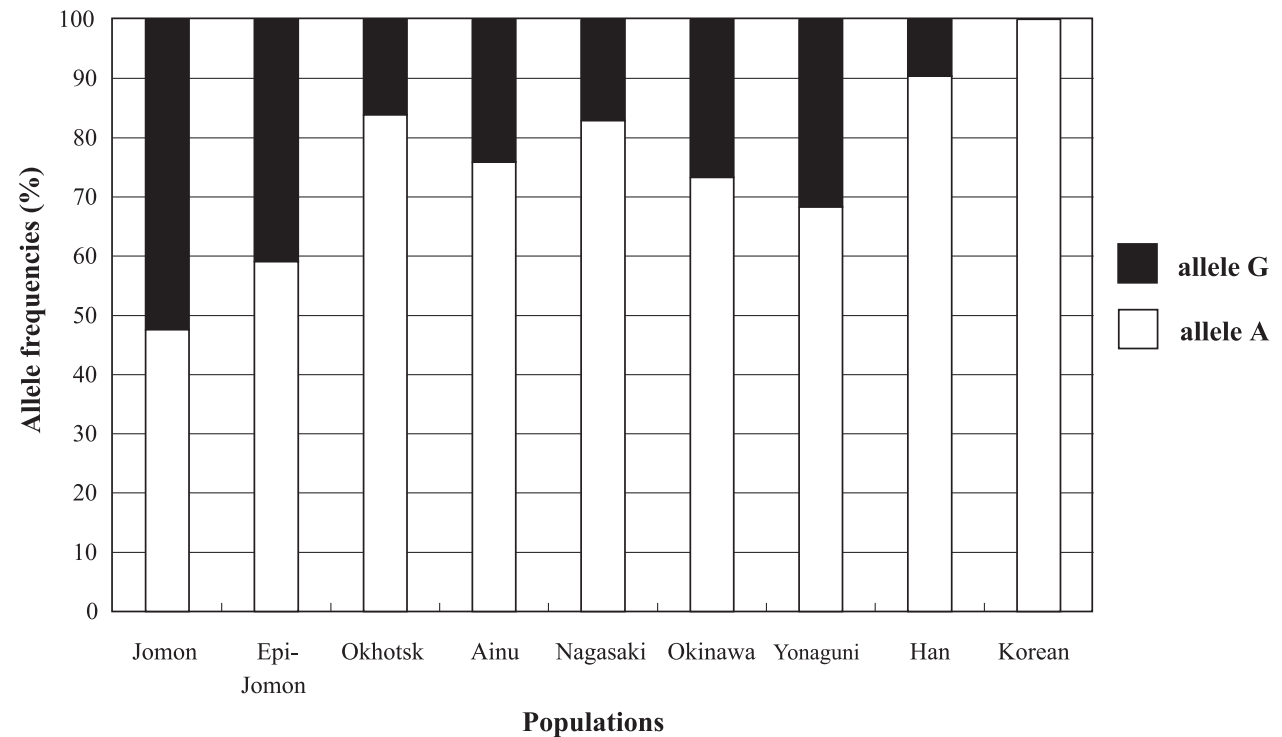

Figure 2. Allele frequencies of the $A B C C 11$ gene among East Asian populations. Frequencies of the Jomon and Epi-Jomon people are shown as data combining the present study and Sato et al. (2009b). Data of the Okhotsk people are cited from Sato et al. (2009b); those of the Ainu, Nagasaki, Okinawa, Yonaguni, Han Chinese and Korean are cited from Yoshiura et al. (2006). 
Table 3. $P$ values of the exact tests of population differentiation

\begin{tabular}{lccc}
\hline \multirow{2}{*}{ Population } & \multicolumn{3}{c}{$P$ values } \\
\cline { 2 - 4 } & Jomon & Epi-Jomon & Okhotsk \\
\hline Epi-Jomon & 0.45 & $0.03^{* *}$ & \\
Okhotsk & $*$ & 0.12 & 0.26 \\
Ainu & $*$ & & \\
\hline
\end{tabular}

* Significant at $P<0.01$.

** Significant at $P<0.05$.

Satsumon people, who are considered to be direct ancestors of the Ainu. Because haplogroup Y has not been detected from the Epi-Jomon specimens (Adachi et al., 2006, 2009), it has been thought that gene flow might have occurred in the end of the Satsumon period.

The present study, however, show that the allele A frequency in the Epi-Jomon people (0.591) was higher than in the Jomon people (0.476), although the difference was not statistically significant. This difference in allele frequencies between the Jomon and Epi-Jomon people might have been caused by slight genetic influences of population(s), such as the Okhotsk people, who possessed a high frequency of allele A, or genetic drift in Hokkaido after the Jomon period.

A morphological study reported that the diversity of the Epi-Jomon people markedly increased from that of the Jomon people (Ishida, 1999); however, the exact test of population differentiation showed that $A B C C 11$ allele frequencies between the Jomon and Epi-Jomon people were not significantly different $(P>0.05)$ (Table 3$)$. Because this non-significant $P$ value may have been caused by the small number of Epi-Jomon specimens successfully analyzed in the present study or the slight difference in allele frequencies between the Jomon and Epi-Jomon people, further investigation of the $A B C C 11$ gene for additional Epi-Jomon specimens is necessary to clarify the allele frequency in the larger gene pool of the Epi-Jomon people. Masuda et al. (2001) reported that some juvenile (less than one year old) ancient brown bears (Ursus arctos) excavated from the archaeological site of the Okhotsk culture on Rebun Island of northern Hokkaido could have originated from southern Hokkaido occupied by the Epi-Jomon culture. In addition, Masuda et al. (2001) showed the intercultural association between the Okhotsk people of Rebun Island and the Epi-Jomon people of southern Hokkaido through the usage of juvenile bears for the bear-sending ceremony; therefore, genetic exchanges between the Epi-Jomon and Okhotsk people might have also occurred.

Otherwise, the results of the present study suggest that Yayoi immigrants to southern Hakkaido might have slightly affected genetically the Jomon people native to Hokkaido. In fact, Matsumura (2001) examined the dental characteristics of the ancient people of the Japanese archipelago, and reported that several Epi-Jomon specimens in southern Hokkaido were classified as immigrant types of the Yayoi or Okhotsk.

Further analysis of the ancient DNA of the Satsumon and later periods provides direct insight into understanding the anthropological history of northern Japan.

\section{Acknowledgments}

We thank Hiroko Ono (Hokkaido University Museum), Haruto Kodera (Tsurumi University) and Minoru Yoneda (The University of Tokyo) for their helpful comments. This study was supported in part by Grants-in-Aid-for Scientific Research (Nos. 21401023, 22370087, 20-1887) from the Japan Society for the Promotion of Science (JSPS), and a grant from the Suhara Memorial Foundation. The second author (T.S.) was a JSPS Research Fellow.

\section{References}

Adachi N., Sakaue K., Dodo Y., Shinoda K., Umetsu K., Matsumura H., and Ohshima N. (2006) Mitochondrial DNA analysis of the Jomon and Epi-Jomon skeletons in Hokkaido, Japan (second report). DNA Polymorphism, 14: 86-90 (in Japanese).

Adachi N., Shinoda K., Umetsu K., and Matsumura H. (2009) Mitochondrial DNA analysis of Jomon skeletons from the Funadomari site, Hokkaido, and its implication for the origins of Native American. American Journal of Physical Anthropolology, 138: 255-265.

Amano T. (2003a) The Origin of Bear Ceremonialism. Yuzankaku, Tokyo (in Japanese).

Amano T. (2003b) What is the Okhotsk culture? In: Nomura T. and Utagawa Y. (eds.), Epi-Jomon and Okhotsk culture. Hokkaido Shimbun, Sapporo, pp. 110-113 (in Japanese).

Dodo Y. and Kawakubo Y. (2002) Cranial affinities of the EpiJomon inhabitants in Hokkaido, Japan. Anthropological Science, 110: 1-32.

Excoffier L., Laval G., and Schneider S. (2005) Arlequin ver. 3.0: An intergrated software package for population genetics data analysis. Evolutionary Bioinfomatics Online, 1: 47-50.

Horai S., Murayama K., Hayasaka K., Matsubayashi S., Hattori Y., Fucharoen G., Haribara S., Park K.S., Omoto K., and Pan I.H. (1996) Mt DNA polymorphism in East Asian populations, with special reference to the peopling of Japan. American Journal of Human Genetics, 59: 579-590.

Ishida H. (1988) Morphological studies of Okhotsk crania from Omisaki, Hokkaido. Journal of Anthropological Society of Nippon, 96: 17-45.

Ishida H. (1999) Ancient peoples of the North Pacific Rim: Ainu biological relationship with their neighbors. In: Fitshugh W.W. and Dubreuil C.O. (eds.), Ainu: Spirit of a Northern People. University of Washington Press, Washington DC, pp. 52-56.

Kitano T., Yuasa I., Yamazaki K., Nakayashiki N., Miyoshi A., Park K.S., and Umetsu K. (2008) Allele frequencies of a SNP and a 27-bp deletion that are the determinant of earwax type in the $A B C C 11$ gene. Legal Medicine, 10: 113-114.

Komesu A., Hanihara T., Amano T., Ono H., Yoneda M., Dodo Y., Fukumine T., and Ishida H. (2008) Nonmetric cranial variation in human skeleton remains associated with Okhotsk culture. Anthropological Science, 116: 33-47.

Kozintsev A.G. (1990) Ainu, Japanese, their ancestors and neighbors: cranioscopic data. Journal of Anthropological Society of Nippon, 98: 247-267.

Kozintsev A.G. (1992) Prehistoric and recent populations of Japan: multivariate analysis of cranioscopic data. Arctic Anthropology, 29: 104-111.

Masuda R., Amano T., and Ono H. (2001) Ancient DNA analysis of brown bear (Ursus arctos) remains from the archeological site of Rebun Island, Hokkaido, Japan. Zoological Science, 18: 741-751.

Matsumura H. (2001) Differentials of Yayoi immigration to Japan as derived from dental metrics. Homo, 52: 135-156. 
Matsunaga E. (1962) The dimorphism in human normal cerumen. Annals of Human Genetics, 25: 273-286.

Raymond M. and Rousset F. (1995) Genepop version 1.2: population genetic software for exact tests and ecumenicism. Journal of Heredity, 86: 248-249.

Sambrook J., Fritsch E.F., and Maniatis T. (1989) Molecular Cloning: A Laboratory Manual. Cold Spring Harbor Laboratory Press, Cold Spring Harbor, NY.

Sato T., Amano T., Ono H., Ishida H., Kodera H., Matsumura H., Yoneda M., and Masuda R. (2007) Origins and genetic features of the Okhotsk people, revealed by ancient mitochondrial DNA analysis. Journal of Human Genetics, 52: 618627.

Sato T., Amano T., Ono H., Ishida H., Kodera H., Matsumura H., Yoneda M., and Masuda R. (2009a) Mitochondrial DNA haplogrouping of the Okhotsk people based on ancient DNA analysis: an intermediate of gene flow from the continental Sakhalin people to the Ainu. Anthropological Science, 117: 171-180.

Sato T., Amano T., Ono H., Ishida H., Kodera H., Matsumura H., Yoneda M., and Masuda R. (2009b) Allele frequencies of the $A B C C 11$ gene for earwax phenotypes among ancient populations of Hokkaido, Japan. Journal of Human Genetics, 54: 409-413.

Sato T., Kazuta H., Amano T., Ono H., Ishida H., Kodera H.,
Matsumura H., Yoneda M., Dodo Y., and Masuda R. (2010) Polymorphisms and allele frequencies of the $A B O$ blood group gene among the Jomon, Epi-Jomon, and Okhotsk people in Hokkaido, northern Japan, revealed by ancient DNA analysis. Journal of Human Genetics, 55: 691-696.

Utagawa Y. (2002) The world of the Okhotsk "bear festival." In: Nishiaki Y. and Utagawa Y. (eds.), Another World of the North. The University of Tokyo Press, Tokyo, pp. 106-113 (in Japanese).

Yamaguchi B. (1974) Paleoanthoropology in Hokkaido. The Quaternary Research, 12: 257-264 (in Japanese).

Yamaguchi B. (1981) Human skeletal remains in Hokkaido. In: Ogata T. (ed.), Japanese I. Anthropology 5. Tokyo, Yuzankaku, pp. 137-156 (in Japanese).

Yoshiura K., Kinoshita A., Ishida T., Ninokata A., Ishikawa T., Kaname T., Bannai M., Tokunaga K., Sonoda S., Komaki R., Ihara M., Saenko V.A., Alipov G.K., Sekine I., Komatsu K., Takahashi H., Nakashima M., Sosonkina N., Mapendano C.K., Ghadami M., Nomura M., Liang D.S., Miwa N., Kim D.K., Garidkhuu A., Natsume N., Ohta T., Tomita H., Kaneko A., Kikuchi M., Russomando G., Hirayama K., Ishibashi M., Takahashi A., Saitou N., Murray J.C., Saito S., Nakamura Y., and Niikawa N. (2006) A SNP in the $A B C C 11$ gene is the determinant of human earwax type. Nature Genetics, 38: 324-330. 